\title{
Correction to: Computing Importance Value of Medical Data Parameters in Classification Tasks and Its Evaluation Using Machine Learning Methods
}

\author{
Andrea Peterkova, Martin Nemeth, German Michalconok, \\ and Allan Bohm
}

\section{Correction to: \\ Chapter "Computing Importance Value of Medical Data Parameters in Classification Tasks and Its Evaluation Using Machine Learning Methods" in: R. Silhavy (Ed.): Software Engineering and Algorithms in Intelligent Systems, AISC 763, https://doi.org/10.1007/978-3-319-91186-1_41}

In the original version of the book, acknowledgement should be included in chapter "Computing Importance Value of Medical Data Parameters in Classification Tasks and Its Evaluation Using Machine Learning Methods". The correction chapter and the book have been updated with the change. 